\title{
Osteogénesis imperfecta (OI) en una mujer adulta y su hija: reporte de caso
}

\author{
Juan Camilo Sarmiento Ramón ${ }^{1}$; Juan Carlos Rojas Castillo ${ }^{1}$; Edwin Antonio Wandurraga \\ Sánchez $z^{2}$; Gustavo Adolfo Parra Serrano ${ }^{2}$; Juan Guillermo Sarmiento Ramón ${ }^{2}$
}

${ }^{1}$ Médico General. Universidad Autónoma de Bucaramanga. FOSCAL Internacional. Endoriente.

${ }^{2}$ Médico Endocrinólogo. Profesor Universidad Autónoma de Bucaramanga.

FOSCAL Internacional. Endoriente.

Institución: Universidad Autónoma de Bucaramanga. FOSCAL Internacional. Endoriente.

Correspondencia: Juan Camilo Sarmiento Ramón

Correo electrónico: jsarmiento19@unab.edu.co, juancsar-

miento19@gmail.com

Dirección postal: Calle 158 \# 20 - 95. FOSCAL Internacional. Endoriente. Consultorio 408. Teléfono: +57-(7)6914813, +57(312) 3571675

Declaración de fuentes de financiación y posibles conflictos de interés: No requirió financiación. No presentamos conflictos de interés. Este reporte de caso fue presentado en modalidad póster en el VIII Congreso de ACOMM en marzo de 2015 y en el Congreso ENDO (Endocrine Society) en marzo de 2015.

Fecha de recepción: $23 / 04 / 2016$

Fecha de aceptación: 5/07/2016

\section{Resumen}

$\mathrm{L}$ a osteogénesis imperfecta (OI) corresponde a un conjunto de trastornos hereditarios del tejido conectivo que tienen como manifestación común la fragilidad ósea. Su etiología es de origen genético y la gran mayoría de casos corresponden a mutaciones autosómicas dominantes de genes que codifican para el colágeno tipo I. Su diagnóstico es primariamente clínico basado en las características típicas de la enfermedad. Reportamos el caso de una mujer con historia de fracturas recurrentes en diferentes ocasiones, y el de su hija de tres años, quien al momento del reporte ya ha presentado dos fracturas.

Palabras clave: osteogénesis imperfecta, osteoporosis, fracturas óseas, adulto, terapia.

\section{Abstract}

Osteogenesis imperfecta (OI) encompasses a group of inherited connective tissue with bone fragility as its common manifestation. Its etiology is genetic in nature and the vast majority of cases are due to autosomal dominant mutations of genes that code for collagen type I proteins. Diagnosis is primarily based on the typical clinical features of the disease. We report the case of a woman with a history of recurrent fractures at different moments in time, and her three year old daughter who at the momento of this report has already had two fractures.

Keywords: osteogenesis imperfecta, osteoporosis, bone fractures, adult, therapeutics.

\section{Introducción}

La osteogénesis imperfecta (OI) es un trastorno hereditario heterogéneo del tejido conectivo caracterizado por fragilidad y baja densidad ósea. Su prevalencia es de aproximadamente uno de cada 20.000 nacidos vivos ${ }^{(1)}$ y sus características clínicas típicas incluyen: fracturas atípicas o excesivas, escleras azules, dentinogénesis imperfecta (DI), pérdida de la audición, escoliosis, hiperlaxitud de ligamentos y piel y deformidades de huesos craneales. Sin embargo, su espectro de presentación clínica es muy amplio, variando desde letalidad in utero hasta formas clínicas leves sin fracturas. Esta variabilidad extrema en la presentación de los pacientes con OI está dada por su heterogeneidad genética y bioquímica ${ }^{(2)}$. Más de un $90 \%$ de casos de OI están asociados con mutaciones autosómicas dominantes en los genes que codifican para las cadenas alfa del colágeno tipo I (genes COL1A1 y COL1A2). Sin embargo, en la última década se han descubierto múltiples genes adicionales involucrados en la modificación postraduccional del colágeno tipo I, la señalización intracelular ósea y la regulación de la homeostasis de matriz ósea, lo que ha expandido el espectro clínico de la $\mathrm{OI}^{(3-7)}$ (tabla 1).

El diagnóstico de OI es usualmente clínico en pacientes que presentan las características clínicas típicas descritas anteriormente o en individuos con historia familiar de $\mathrm{OI}^{(3,8)}$. Sin embargo, en ausencia de dichas condiciones, el diagnóstico puede representar un reto clínico importante; ya que en formas leves de la enfermedad, el único hallazgo positivo puede ser la disminución de la densidad mineral ósea sin presencia de fracturas o de manifestaciones extraesqueléticas. En la actualidad no hay una prueba diagnóstica definitiva para OI. 
Tabla 1. Mutaciones autosómicas dominantes en osteogénesis imperfecta (OI)

\begin{tabular}{c|c|c|c|l}
\hline Condición & Gen & Mecanismo de enfermedad & Severidad & \multicolumn{1}{|c}{ Características asociadas } \\
Ol tipo I & COL1A1 & $\begin{array}{c}\text { Disminución de la producción } \\
\text { de colágeno tipo I }\end{array}$ & $\begin{array}{l}\text { No deformante } \\
\text { Fracturas prepuberales } \\
\text { Insuficiencia aórtica }\end{array}$ \\
\hline Ol tipo II & $\begin{array}{l}\text { COL1A1 } \\
\text { COL1A2 }\end{array}$ & $\begin{array}{c}\text { Producción anormal de } \\
\text { colágeno tipo I }\end{array}$ & Letal & $\begin{array}{l}\text { Hipoplasia pulmonar } \\
\text { Malformaciones del SNC }\end{array}$ \\
\hline Ol tipo III & $\begin{array}{l}\text { COL1A1 } \\
\text { COL1A2 }\end{array}$ & $\begin{array}{c}\text { Producción anormal de } \\
\text { colágeno tipo I }\end{array}$ & $\begin{array}{l}\text { Talla baja } \\
\text { Facies triangulares } \\
\text { Deformidades de huesos largos }\end{array}$ \\
\hline Ol tipo IV & $\begin{array}{c}\text { COL1A1 } \\
\text { COL1A2 }\end{array}$ & $\begin{array}{c}\text { Producción anormal de } \\
\text { colágeno tipo I }\end{array}$ & Moderada & $\begin{array}{l}\text { Talla baja } \\
\text { Escoliosis } \\
\text { Hiperlaxitud ligamentaria }\end{array}$ \\
\hline Ol tipo V & IFITM5 & $\begin{array}{c}\text { Desregulación de la } \\
\text { mineralización del colágeno }\end{array}$ & Moderada & $\begin{array}{l}\text { Calcificación de membrana intraóseas } \\
\text { Dislocación de cabeza del radio }\end{array}$ \\
\hline
\end{tabular}

Adaptada de: Harrington J, Sochett E, Howard A. Update on the evaluation and treatment of osteogenesis imperfecta. Pediatric Clin North Am. 2014 Dec;61(6):1243-57. doi: 10.1016/j.pcl.2014.08.010. Epub 2014 Sep 22

Sin embargo, hay herramientas disponibles para determinar la estructura y cantidad del colágeno tipo I in vitro utilizando cultivos de fibroblastos obtenidos por biopsias de piel. Una anormalidad del colágeno tipo I está presente en al menos el $90 \%$ de casos de $\mathrm{OI}^{(5,6)}$. Adicionalmente, están disponibles pruebas de ADN para detección de mutaciones en los genes COL1A1 y COL1A2, los cuales detectan más del $90 \%$ de casos de OI. A pesar de contar con dichos análisis genéticos, estudios negativos no descartan la presencia de enfermedad, debido a que aproximadamente un $10 \%$ de casos están relacionados con mutaciones diferentes a las asociadas al colágeno tipo $\mathrm{I}^{(7)}$.

El manejo de pacientes con OI tiene como objetivos principales reducir la tasa de fracturas, prevenir las deformidades de huesos largos y columna vertebral, minimizar el dolor y maximizar la movilidad y calidad de vida del paciente ${ }^{(8,9)}$. En la actualidad, los bifosfonatos son el pilar del tratamiento farmacológico para la mayoría de formas de OI y corresponden al grupo de medicamentos más estudiado y utilizado como opción terapéutica en la enfermedad ${ }^{(10,11)}$. Los bifosfonatos han demostrado aumentar la densidad mineral ósea (DMO) en niños y adultos, mientras que los datos sobre la incidencia de fracturas son contradictorios ${ }^{(12-15)}$. A pesar de la falta de evidencia más contundente, los bifosfonatos son ampliamente utilizados en OI para la prevención de fracturas, aunque para determinar la dosificación y duración ideal del tratamiento aún se requieren estudios adicionales.

\section{Presentación de casos}

\section{Caso \# 1}

Mujer de 26 años con cuadro clínico consistente en múltiples fracturas a lo largo de su vida con inicio a los 17
Figura 1. Fractura de fémur izquierdo

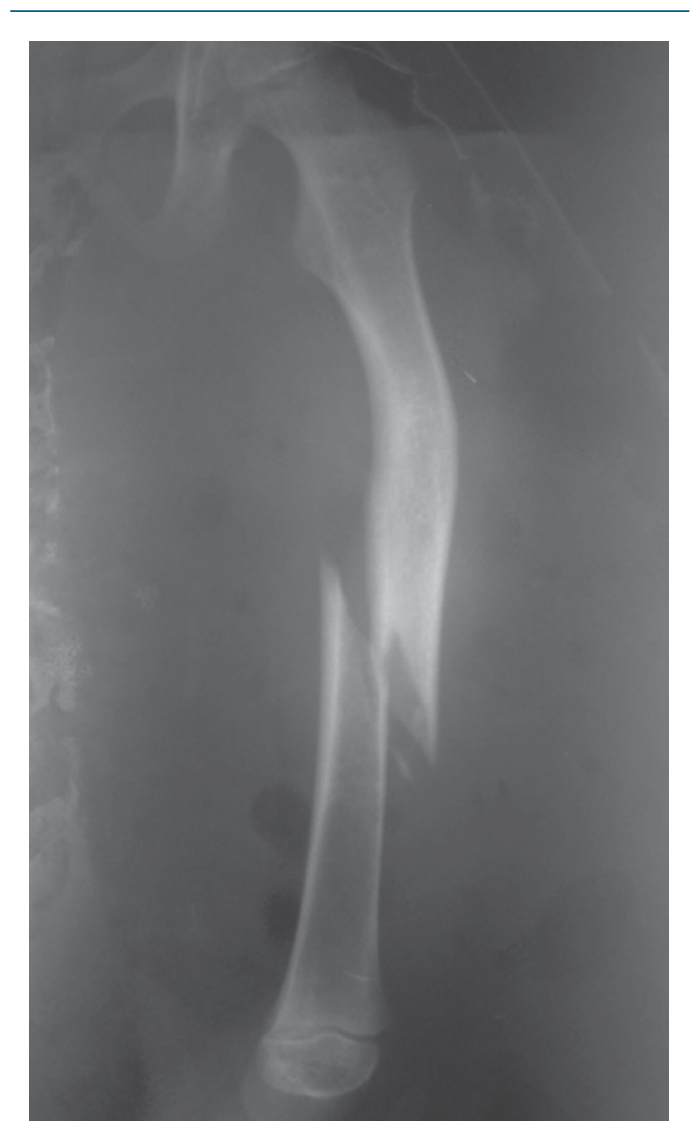

meses de edad con fractura de fémur izquierdo y refractura del mismo a los 4 años de edad (figura 1), posteriormente a los 9 años fractura condílea derecha y a los 10 años fractura radial y cubital izquierda (figura 2), todas con traumas míni- 
Figura 2. Fractura de radio y cúbito izquierdos

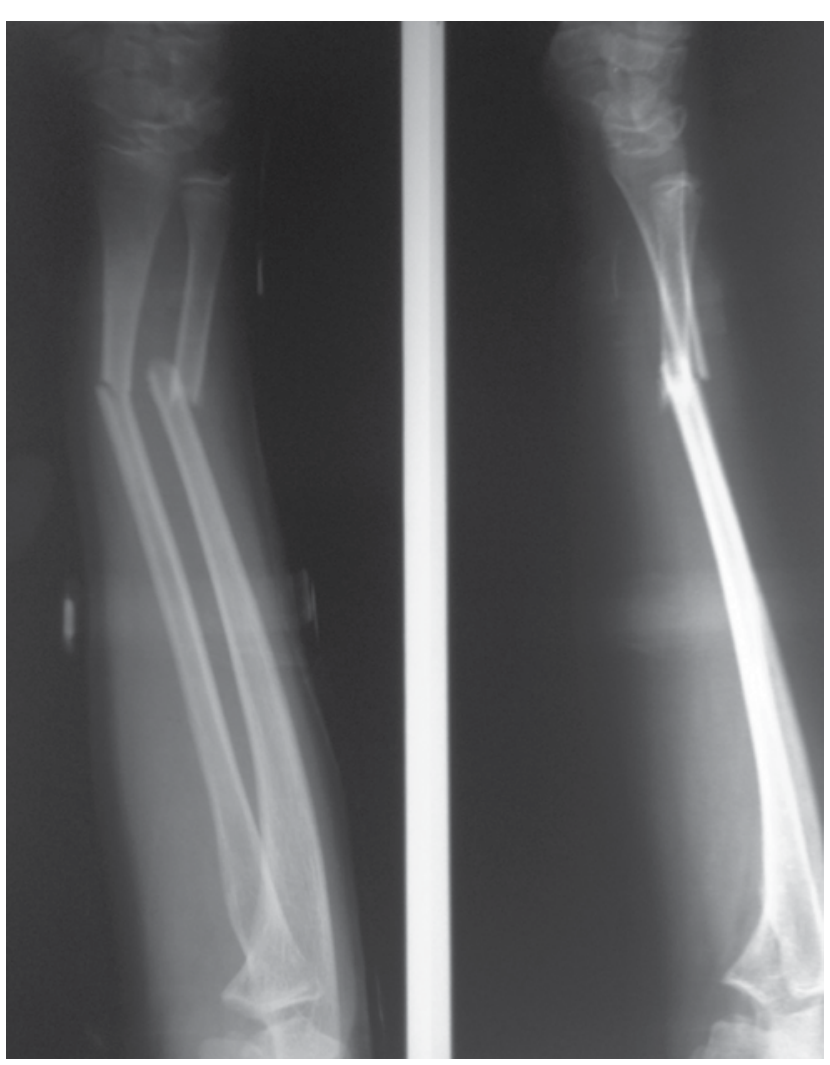

Figura 3. Escleras azules en paciente madre

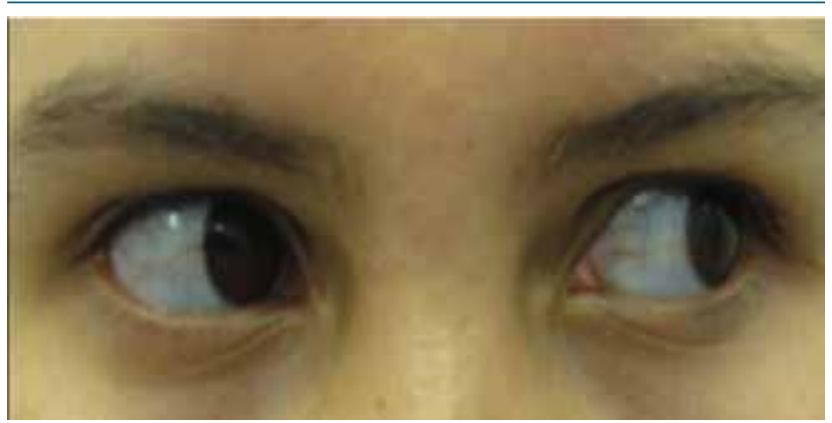

mos. Al examen físico peso: $70 \mathrm{~kg}$, talla: $154 \mathrm{~cm}$, IMC: 29,52, escleras azules (figura 3), sin alteración de la dentición ni de la agudeza auditiva, clinodactilia de dedos de la mano derecha y braquidactilia de dedos de los pies. En los estudios radiológicos se evidenció osteopenia. Densitometría ósea central (DXA) muestra $\mathrm{Z}$ score a nivel de columna lumbar L2-L4 (se excluye L1) de - 4,1 DE, con una densidad mineral ósea de 0,703 gr/ $\mathrm{cm}^{3}$, indicativo de baja masa ósea para la edad. Se realizan estudios de osteoporosis secundaria (tabla 2) documentando insuficiencia de vitamina $\mathrm{D}$, ligero aumento de la fosfatasa alcalina y marcadores de resorción en orina (Pirilinks -Deoxipiridinolina) muy elevados. Gammagrafía ósea evidencia deformidad en varo en ambos fémur; hipercaptación cortical media y lateral en huesos largos; fracturas postraumáticas a nivel de
Tabla 2. Laboratorios iniciales caso 1

\begin{tabular}{c|c|c}
\hline Paraclínico & Resultado & Valor referencia \\
\hline $25 \mathrm{OH}$ vitamina D3 & $29 \mathrm{ng} / \mathrm{ml}$ & $30-100 \mathrm{ng} / \mathrm{ml}$ \\
\hline Calcio & $9,3 \mathrm{mg} / \mathrm{dl}$ & $8,4-10,2 \mathrm{mg} / \mathrm{dl}$ \\
\hline Fosfatasa alcalina & $149 \mathrm{u} / \mathrm{l}$ & $35-104 \mathrm{u} / \mathrm{l}$ \\
\hline Fósforo & $3,2 \mathrm{mg} / \mathrm{dl}$ & $2,7-4,5 \mathrm{mg} / \mathrm{dl}$ \\
\hline $\begin{array}{c}\text { Cortisol en orina } 24 \\
\text { horas }\end{array}$ & $25,3 \mathrm{mcg} / 24 \mathrm{~h}$ & $20-130 \mathrm{ug} / 24 \mathrm{~h}$ \\
\hline PTH intacta & $46,45 \mathrm{pg} / \mathrm{ml}$ & $15-65 \mathrm{pg} / \mathrm{ml}$ \\
\hline TSH & $1,18 \mathrm{mcUl} / \mathrm{mL}$ & $0,27-4,20 \mathrm{mcUl} / \mathrm{mL}$ \\
\hline Pirilinks & 12,8 & $3,0-7,4$ \\
\hline Glicemia basal & $86 \mathrm{mg} / \mathrm{dl}$ & $74-106 \mathrm{mg} / \mathrm{dl}$ \\
\hline Calcio orina $24 \mathrm{~h}$ & $29,15 \mathrm{mg} / 24 \mathrm{~h}$ & $100-321 \mathrm{mg} / 24$ \\
\hline Estradiol & 61.56 & $12,5-166$ \\
\hline FSH & $5,22 \mathrm{mUl} / \mathrm{ml}$ & $4,7-21,5 \mathrm{mUl} / \mathrm{ml}$ \\
\hline LH & $16,9 \mathrm{Ul} / \mathrm{L}$ & \\
\hline Prolactina & $11,5 \mathrm{ng} / \mathrm{ml}$ & $2-25 \mathrm{ng} / \mathrm{ml}$ \\
\hline
\end{tabular}

base de todos los metatarsianos del pie izquierdo, y primer y quinto metatarsiano del pie derecho. El diagnóstico fue confirmado por medio de estudios genéticos que revelaron una mutación en estado heterocigoto en exón 19: c.1299+1G>A en sitio de empalme del gen COL1A1, lo que llevó a un diagnóstico final de osteogénesis imperfecta (OI) tipo I. Actualmente se encuentra en manejo con ácido zoledrónico endovenoso sin presentar nuevas fracturas. A pesar de las recomendaciones, la paciente queda en estado de embarazo. Asiste a consulta con su hija de 14 meses quien presenta escleras azules.

\section{Caso \# 2}

Lactante mayor de 17 meses de edad, sexo femenino, producto de cesárea, nacida a término, edad gestacional de 37 semanas, peso: $3.090 \mathrm{~kg}$, talla $50 \mathrm{~cm}$, apgar $8 / 10$ a los 5 minutos, 10/ 10 a los 10 minutos. Al examen físico realizado se evidencian escleras azules (figura 4), resto de examen físico normal. Paciente con adecuado desarrollo pondoestatural y psicomotor, no se evidencia aspecto sindrómico ni rasgos dismórficos.

Figura 4. Escleras azules en paciente hija

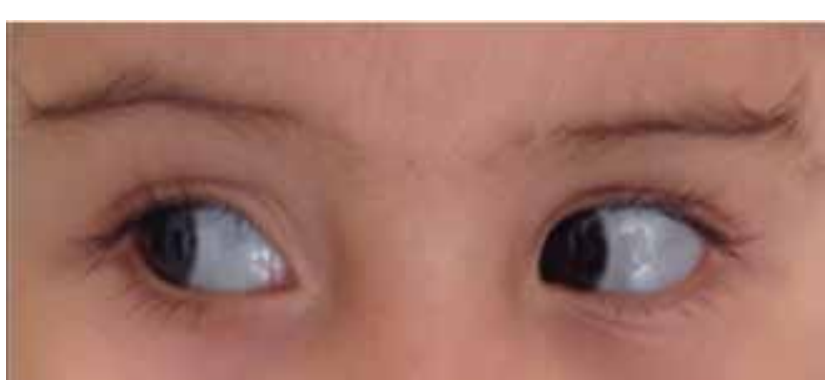


Durante el primer año de edad presenta primera fractura a nivel de peroné izquierdo posterior a caída desde su propia altura mientras se encontraba en el desarrollo primario de la deambulación. A los 16 meses de edad, fractura de húmero izquierdo. Debido al antecedente de madre con OI se envía estudio molecular por genética el cual revela la misma mutación evidencia en su madre.

\section{Discusión}

La osteogénesis imperfecta (OI) es un conjunto de trastornos hereditarios del tejido conectivo con una amplia heterogeneidad en su severidad, pero que tienen como característica común la presencia de fragilidad ósea ${ }^{(8)}$. Los pacientes con este trastorno sufren de múltiples fracturas asociadas a traumas mínimos, lo que le ha atribuido el término de la "enfermedad de los huesos de cristal" ${ }^{(1,8)}$. Los pacientes diagnosticados con OI deben ser cuidadosamente examinados y aconsejados con el fin de evitar lesiones y fracturas recurrentes. Adicionalmen- te es importante reconocer su modo de transmisión genética y discutir claramente con los padres las posibilidades de que su hijo o hija presenten igualmente dicha condición. El tratamiento debe ser multidisciplinario y debe incluir un acercamiento no farmacológico que incluye terapias ocupacionales y físicas con el fin de asistir en el desarrollo motor grueso y la independencia funcional del paciente ${ }^{(1,8,9)}$. Dentro del tratamiento farmacológico, los bifosfonatos son ampliamente utilizados con el objetivo de incrementar la mineralización ósea y disminuir el número de fracturas ${ }^{(10-15)}$. En pacientes que ya han presentado fracturas, el tratamiento se basa en la estabilización quirúrgica con el fin de recuperar la función motora y corregir deformidades.

A pesar de ser rara y con presentaciones clínicas muy variables, se debe sospechar la presencia de osteogénesis imperfecta (OI) en pacientes que acuden a un servicio médico con historia de múltiples fracturas, densidad mineral ósea baja y escleras azules; especialmente en aquellos con historia familiar de la enfermedad.

\section{Referencias}

1. Harrington J, Sochett E, Howard A. Update on the evaluation and treatment of osteogenesis imperfecta. Pediatric Clin North Am. 2014 Dec;61(6):124357. doi: 10.1016/j.pcl.2014.08.010. Epub 2014 Sep 22.

2. Sillence DO, Senn A, Danks DM. Genetic heterogeneity in osteogenesis imperfecta. J Med Genet 1979;16(2):101-16.

3. Van Dijk FS, Sillence DO. Osteogenesis imperfecta: clinical diagnosis, nomenclature and severity assessment. Am J Med Genet A. 2014;8(10):36545.

4. Ben Amor IM, Glorieux FH, Rauch F. Genotype-phenotype correlations in autosomal dominant osteogenesis imperfecta. J Osteoporos. 2011;2011:540178.

5. Bodian DL, Chan TF, Poon A, et al. Mutation and polymorphism spectrum in osteogenesis imperfecta type II: implications for genotype-phenotype relationships. Hum Mol Genet 2009;18(3):463-71.

6. Willing MC, Deschenes SP, Scott DA, et al. Osteogenesis imperfecta type I: molecular heterogeneity for COL1A1 null alleles of type I collagen. Am J Hum Genet 1994;55(4):638-47.

7. Körkkö J, Ala-Kokko L, De Paepe A, Nuytinck L, Earley J, Prockop DJ. Analysis of the COL1A1 and COL1A2 genes by PCR amplification and scanning by conformation-sensitive gel electrophoresis identifies only COL1A1 mutations in 15 patients with osteogenesis imperfecta type I: Identification of common sequences of null-allele mutations. Am J Hum Genet. 1998;62(1):98.
8. Rauch F, Glorieux FH. Osteogenesis imperfecta. Lancet. 2004;363(9418):1377.

9. Engelbert RH, Beemer FA, van der Graaf Y, Helders PJ. Osteogenesis imperfecta in childhood: impairment and disability: a follow-up study. Arch Phys Med Rehabil. 1999;80(8):896.

10. She CG, Zhang Y, Yuan W. Efficacy of biphosphonates on bone mineral density and fracture rates in patients with osteogenesis imperfecta: A systematic review and meta-analysis. Am J They. 2015 Apr 3.

11. Hald JD, Evangelou E, Langdahl BL, Ralston SH. Biphosphonates for the prevention of fractures in osteogenesis imperfecta: meta-analysis of placebocontrolled trials. J Bone Miner Res. 2015 May;30(5):929-33. dos: 10.1002/ jbmr.2410.

12. Falk MJ, Heeger S, Lynch A. Intravenous bisphosphonate thera- py in children with osteogenesis imperfect. Pediatrics 2003; 111: 573-8.

13. Bajpai A, Kabra M , Gupta N, Sharda S, Ghosh M. Intravenous pamidronate therapy in osteogenesis imperfecta: response to treatment and factors in uencing outcome. J Pediatr Orthop 2007; 27: 225-7.

14. Lazala 0 , Solaque H. Terapia con bisfosfonatos en osteogenesis imperfecta. Rev Col Or Tra. 2009;23(2):109-14.

15. Sánchez-Sánchez LM, Cabrera-Pedroza AU, Palacios-Saucedo G, et al. Ácido zoledrónico (zolendronato) en niños con osteogénesis imperfecta (OI). Gac Med Mex. 2015;151:164-8. 